Pacific Journal of Mathematics

GROUPS OF ISOMETRIES OF A TREE AND THE 


\section{GROUPS OF ISOMETRIES OF A TREE AND THE KUNZE-STEIN PHENOMENON}

\section{Claudio Nebbia}

In this paper we prove that every group of isometries of a homogeneous or semihomogeneous tree which acts transitively on the boundary of the tree is a Kunze-Stein group. From this, we deduce a weak Kunze-Stein property for groups acting simply transitively on a tree (in particular free groups on finitely many generators).

1. Introduction. Let $G$ be a locally compact group, then $G$ is said to satisfy the "Kunze-Stein property" or sometimes $G$ is called a "KunzeStein group" if $L^{p}(G) * L^{2}(G) \subset L^{2}(G)$ for every $1<p<2$.

This property was discovered by R. A. Kunze and E. M. Stein for the group $\mathrm{SL}_{2}(\mathbf{R})$ [15]. Later the same property was proved for every connected semisimple Lie group with finite center by M. Cowling [6]. In this paper we prove that every locally compact group of isometries of a homogeneous or semihomogeneous tree has the Kunze-Stein property provided that $G$ acts transitively on the boundary of the tree. The proof of our Theorem is based on M. Cowling's proof of the KunzeStein phenomenon for $\mathrm{SL}_{2}(\mathbf{R})$ [6]. A weaker property is deduced for discrete groups acting simply transitively on the tree but not on the tree boundary.

It is known that the group $\mathrm{SL}_{2}(\kappa)$, where $\kappa$ is a local field, may be realized as a closed subgroup of the group of all isometries of a homogeneous tree in such a way that $\mathrm{SL}_{2}(\kappa)$ acts transitively on the boundary [17]. In particular our result implies that $\mathrm{SL}_{2}(\kappa)$ is a KunzeStein group for every local field. This was proved by Gulizia [13] for a local field $\kappa$ such that the finite residue class field associated with $\kappa$ is not of characteristic 2.

We follow the terminology and definitions of [6]. In particular $A(G)$ is the Fourier algebra of $G$ as defined in [7]; $C_{00}(G)$ denotes the space of continuous functions with compact support and $L^{p}(G), 1 \leq p \leq$ $\infty$, the usual $L^{p}$-space with respect to a fixed left Haar measure. As observed in [6], a locally compact group $G$ is a Kunze-Stein group if and only if $A(G) \subset L^{q}(G)$ for every $q>2$. We will also use the theory of representations for groups acting on a tree developed by P. Cartier 
[3], A. Figà-Talamanca and M. A. Picardello [11, 12]. A convenient reference is [12]. In fact the results we quote and use from [12] are all valid with essentially the same proof when a discrete group acting simply transitively on a tree replaces the free group [1].

I wish to thank A. Figà-Talamanca for his encouragement during the preparation of this paper. I would also like to thank Prof. G. Rousseau for bringing reference [2] to my attention.

2. Notations. We shall give a concise description of the tree and of the group of isometries. We refer the reader to $[3,17,18]$ for undefined notions and terminology. Let $X$ be a homogeneous tree of order $r$; the distance $d(x, y)$ is defined as the length of the unique geodesic $[x, y]$ connecting $x$ to $y$. Let $\operatorname{Aut}(X)$ be the group of all isometries of $X$. We assume also $r \geq 3$ (otherwise, for $r=2, \operatorname{Aut}(X)$ is amenable and noncompact, hence it is not a Kunze-Stein group). $\operatorname{Aut}(X)$ is a locally compact separable group and the stability subgroup $K$ of a vertex of $X$ is compact and open in $\operatorname{Aut}(X)$. A subgroup $\Gamma$ of $\operatorname{Aut}(X)$ is called simply transitive if it acts transitively on the vertices and $\Gamma \cap K=\{1\}$. In other words, $\Gamma$ acts simply transitively on $X$ iff the map $\gamma \in \Gamma \rightarrow \gamma\left(x_{0}\right) \in X$ is bijective for a fixed vertex $x_{0}$ in $X$. It is known that every such group is isomorphic to the free product of $t$ copies of the integers and $s$ copies of the group of order 2 with $2 t+s=r[1,4]$. Since $K$ is open, $\Gamma$ is discrete in $\operatorname{Aut}(X)$. Moreover $\Gamma \cdot K=\operatorname{Aut}(X)$ and $\Gamma$ is a lattice. As usual, let $\langle f, h\rangle=\int f(g) h(g) d g$.

Let $\Omega$ be the boundary of the tree, that is the set of equivalence classes of sequences of distinct vertices $\left\{s_{n}: n=0,1,2, \ldots\right\}$ such that $d\left(s_{i}, s_{i+1}\right)=1$ for every $i=0,1,2, \ldots$; two such sequences are said to be equivalent if they have infinitely many common vertices.

$\Omega$ is a compact metric space; if $x_{0} \in X$ and $\omega_{0} \in \Omega$ there exists a unique sequence of distinct vertices $\left\{s_{n}\right\}$ in the class $\omega_{0}$ such that $s_{0}=x_{0}$. In this way, $\Omega$ can be regarded as the set of infinite sequences starting from a fixed vertex $x_{0}$ in $X$. There exists a unique probability measure $\nu$ on $\Omega$, Aut $(X)$-quasi invariant and $K$-invariant. Let $P(g, \omega)$ be the Poisson kernel, that is, for $g \in \operatorname{Aut}(X)$ and $\omega \in \Omega, P(g, \omega)=$ $d \nu_{g} / d \nu(\omega)$, with $\nu_{g}(\omega)=\nu\left(g^{-1} \omega\right)$.

For every complex number $z$, we define the following representation of $\operatorname{Aut}(X)$ :

$$
\left[\pi_{z}(g) f\right](\omega)=P^{z}(g, \omega) f\left(g^{-1} \omega\right)
$$


It is known that, for $t \in \mathbf{R}, \pi_{1 / 2+i t}$ are unitary irreducible representations on $L^{2}(\Omega)$; in fact even the restrictions to $\Gamma$ are irreducible [12, pg. $76 ; 1]$.

For a fixed vertex $x_{0}$ in $X$, let $X^{+}=\left\{x \in X: d\left(x, x_{0}\right)\right.$ is even $\}$ and $X^{-}=X \backslash X^{+}$. The partition $X^{+}, X^{-}$is independent of the choice of $x_{0}$. If $G$ is a closed unimodular subgroup of $\operatorname{Aut}(X)$ acting transitively on $X^{+}$but not on the tree, then the representations $\left.\pi_{1 / 2+i t}\right|_{G}$ are irreducible for $t \neq(2 m+1) \pi / 2 \lg (r-1), m \in Z$ [2, pg. 39, pg. 62]. Let $J$ be the interval $[0, \pi / \lg (r-1)]$ and $c(z)$ the following complex function:

$$
c(z)=\left[(r-1)^{2-2 z}-1\right] /\left[(r-1)^{1-2 z}-1\right] .
$$

Finally, let $d m$ be the following measure:

$$
d m(t)=\left[(r-1) \lg (r-1) / 4 \pi r\left|c\left(\frac{1}{2}+i t\right)\right|^{2}\right] d t .
$$

3. The results. Let $G$ be a closed noncompact subgroup of $\operatorname{Aut}(X)$ acting transitively on $\Omega$, and $K_{0}=G \cap K$. Since $K_{0}$ is compact open in $G$ we can assume that its measure is one.

\section{Proposition 1. $K_{0}$ acts transitively on $\Omega$.}

Proof. Since $G / K_{0}$ is countable, Baire's theorem implies that every orbit of $K_{0}$ on $\Omega$ is open. By [17, Prop. 3.4], there exist $g \in G$, a sequence $\left\{s_{n}\right\} \subset X, n \in Z$ and $i_{0} \in Z i_{0} \neq 0$, such that $d\left(s_{n}, s_{n+1}\right)=1$ and $g\left(s_{n}\right)=s_{n+i_{0}}$ for every $n \in Z$. In this proof we realize $\Omega$ as the set of all infinite sequences $\left\{t_{n}\right\}$ issued from $t_{0}=s_{0}$. Therefore the sets: $E(x)=\left\{\left\{t_{n}\right\} \in \Omega: t_{j}=x\right\}$ with $x \in X$ and $d\left(s_{0}, x\right)=j$ form a basis for the topology of $\Omega$. Let $\omega_{1}=\left\{s_{0}, s_{1}, \ldots\right\}$ and $\omega_{2}=\left\{s_{0}, s_{-1}, s_{-2}, \ldots\right\}$.

Since $K_{0} \omega_{1}$ and $K_{0} \omega_{2}$ are open, it follows that there exists $j>0$ such that $E\left(s_{j}\right) \subset K_{0} \omega_{1}$ and $E\left(s_{-j}\right) \subset K_{0} \omega_{2}$. Using the automorphism $g$, it is not hard to show that $K_{0}$ acts transitively on $\complement E\left(s_{-1}\right)$ and $\complement E\left(s_{1}\right)$, respectively. Obviously, $\complement E\left(s_{-1}\right) \cap \complement E\left(s_{1}\right) \neq \varnothing$ and $\complement E\left(s_{-1}\right) \cup \complement E\left(s_{1}\right)=$ $\Omega$. This means that $K_{0}$ acts transitively on $\Omega$.

Proposition 2. Let $G$ be a closed noncompact subgroup of $\operatorname{Aut}(X)$ acting transitively on $\Omega$. Then either $G$ acts transitively on the vertices of $X$, or $G$ has two orbits $X^{+}$and $X^{-}$.

Proof. By Proposition $1, K_{0}$ acts transitively on $\Omega$, that is, $K_{0}$ acts transitively on the set $S_{n}^{s_{0}}=\left\{y \in X: d\left(s_{0}, y\right)=n\right\}$ for every $n \geq 0$. Moreover for every $g \in G, g K_{0} g^{-1}$ acts transitively on $S_{n}^{x}$ for every $n \geq 0$ and $g\left(s_{0}\right)=x$. In particular for every $x \in G\left(s_{0}\right), G\left(s_{0}\right)$ is an 
infinite union of sets $S_{n}^{x}$. This implies that if $x, y \in G\left(s_{0}\right) d(x, y)=m$, then $S_{m}^{x} \cup S_{m}^{y} \subset G\left(s_{0}\right)$. Therefore $S_{m}^{x} \subset G\left(s_{0}\right)$ implies that:

$$
\bigcup_{j=0}^{+\infty} S_{j m}^{x} \subset G\left(s_{0}\right) \text {. }
$$

If $G\left(s_{0}\right)$ contains vertices $x$ and $y$ with $d(x, y)=1$, then $G\left(s_{0}\right)=X$ and $G$ is transitive on $X$. Suppose now $G\left(s_{0}\right) \neq X$; thus $G\left(s_{0}\right) \subset X^{+}$. Let $t=\min \left\{m>0: S_{m}^{s_{0}} \subset G\left(s_{0}\right)\right\}$. It follows that $G\left(s_{0}\right) \cap S_{m}^{s_{0}}=\varnothing$ for $0<m<2 t m \neq t$ and $\bigcup_{j=0}^{+\infty} S_{j t}^{S_{0}} \subset G\left(s_{0}\right)$. Let $x \in S_{t}^{S_{0}}$ and $\left[s_{0}, x\right]=\left\{s_{0}, x_{1}, x_{2}, \ldots, x_{t-1}, x\right\}$ the geodesic connecting $s_{0}$ to $x$; we can choose $y \in X$ in such a way that $d(y, x)=t, d\left(y, s_{0}\right)=2 t-2$ and $\left[x, s_{0}\right] \cap[x, y]=\left[x, x_{t-1}\right]=\left\{x, x_{t-1}\right\}$. Since $d(x, y)=t, y \in G\left(s_{0}\right)$ but $y \in S_{2 t-2}^{s_{0}}$ so that $S_{2 t-2}^{s_{0}} \subset G\left(s_{0}\right)$. This implies that $2 t-2=t$, that is, $t=2$ and $G\left(s_{0}\right)=X^{+}$. Similarly, we can prove that $G\left(s_{1}\right)=X^{-}$, with $d\left(s_{0}, s_{1}\right)=1$.

The aim of this note is to prove the following Theorem.

THEOREM 1. Every closed subgroup $G$ of $\operatorname{Aut}(X)$ acting transitively on $\Omega$ is a Kunze-Stein group.

It is enough to prove the Theorem for noncompact groups.

First, we observe that:

$$
\int_{J}\left\|\left.\pi_{1 / 2+i t}\right|_{G}(u)\right\|_{H S}^{2} d m(t) \leq\|u\|_{2}^{2} \quad \text { for every } u \text { in } C_{00}(G) .
$$

Indeed $\left(G, K_{0}\right)$ is a Gelfand pair because $K_{0}$ acts transitively on $\Omega$ and $g^{-1} \in K_{0} g K_{0}$ for every $g$ in $G$ [9, Prop. 1.2]. The representations $\left.\pi_{1 / 2+i t}\right|_{G}$ are irreducible iff 1 (the function identically one on $\Omega$ ) is a cyclic vector. By Proposition 2, we have two possibilities: if $G$ is transitive on $X$, then the representations $\left.\pi_{1 / 2+i t}\right|_{G}$ are irreducible for every $t \in J[12$, pg. 76; 1$]$; otherwise for $t \in J, t \neq \pi / 2 \lg (r-1)$ [2, pg. 39, pg. 62].

Since, for Gelfand pairs, the Plancherel measure on the irreducible unitary representations of $G$ having a $K_{0}$-fixed vector depends only on the right $K_{0}$-invariant functions [ 9, Th. $4.2 ; 16, \mathrm{pg}$. 65], to prove the inequality, it is enough to prove that

$$
\int_{J}\left\|\left.\pi_{1 / 2+i t}\right|_{G}(u)\right\|_{H S}^{2} d m(t)=\|u\|_{2}^{2}
$$

for every right $K_{0}$-invariant function $u$ in $C_{00}(G)$. To show this, let $T$ be the following projection on $L^{2}(\Omega): T f=\left[\int_{\Omega} f(\omega) d \nu(\omega)\right] \mathbf{1}$ for 
$f \in L^{2}(\Omega)$. We have $T=\int_{K_{0}} \pi_{1 / 2+i t}(k) d k$ (recall that $K_{0}$ is transitive on $\Omega$ ). Let $\operatorname{Aut}(X)=\Gamma K$; every function $u$ right $K_{0}$-invariant on $G$ corresponds to a function $\tilde{u}$ on $\Gamma$ in such a way that $\|u\|_{2}=\|\tilde{u}\|_{2}$ and $\left.\pi_{1 / 2+i t}\right|_{G}(u)=\left[\left.\pi_{1 / 2+i t}\right|_{\Gamma}(\tilde{u})\right] T$.

Therefore $\left\|\left.\pi_{1 / 2+i t}\right|_{G}(u)\right\|_{H S}=\left\|\left.\pi_{1 / 2+i t}\right|_{\Gamma}(\tilde{u}) \mathbf{1}\right\|_{L^{2}(\Omega)}$; hence the equality follows from $[12, \mathrm{pg} .86 ; 1]$. The proof of Theorem 1 is based on the following two Lemmas.

In the next Lemma, we denote by $G$ a locally compact group and by $L_{1}^{\infty}(G)$ the space of all functions $f$ in $L^{\infty}(G)$ such that $\|f\|_{\infty} \leq 1$; we assume $\phi$ to be a complex continuous function on the strip $S=$ $[\alpha, \beta] \times \mathbf{R}$ with $0<\alpha<\frac{1}{2}<\beta<1$, analytic on $S^{0}=(\alpha, \beta) \times \mathbf{R}$ and such that (1) $\phi$ is bounded on $S$; (2) $|\phi(x+i t)| \geq h(x)>0$ for every $t \in \mathbf{R}$ and $\alpha \leq x \leq \beta, x \neq \frac{1}{2}$. With these notations, we have:

Lemma 1 (M. Cowling [6]). Let $F: S \rightarrow L_{1}^{\infty}(G)$ be a continuous map, analytic on $S^{0}$ (i.e. $\left\langle F_{z}, u\right\rangle$ is an analytic function for every $u$ in $\left.C_{00}(G)\right)$. If there exists a positive constant $c$ such that

$$
\int_{\mathbf{R}}\left|\left\langle F_{1 / 2+i t}, u\right\rangle\right|^{2}\left|\phi\left(\frac{1}{2}+i t\right)\right| d t \leq c\|u\|_{2}^{2} \quad \text { for every } u \text { in } C_{00}(G),
$$

then the function $F_{1 / 2}$ is in $L^{q}(G)$ for every $q>2$.

Proof. This Lemma is obtained from Lemma 2.1 of [6, pg. 215] where $S=[\alpha, \beta] \times \mathbf{R}, q=q^{\prime}=2, X=G$ and $X_{0}$ is a singleton, observing that the function $(z / z-2)^{n}$ could be replaced with a general analytic function $\phi$ with the properties (1) and (2).

LEMMA 2. The coefficients of the quasi-regular representation on $\Omega$, that is the functions:

$$
\left\langle\pi_{1 / 2}(g) \xi, \eta\right\rangle=\int_{\Omega} P^{1 / 2}(g, \omega) \xi\left(g^{-1} \omega\right) \overline{\eta(\omega)} d \nu(\omega)
$$

for $\xi$, $\eta$ in $L^{2}(\Omega)$ and $g$ in $G$

are in $L^{q}(G)$ for every $q>2$.

Proof. Since $\left|\left\langle\pi_{1 / 2}(g) \xi, \eta\right\rangle\right| \leq\left\langle\pi_{1 / 2}(g)|\xi|,|\eta|\right\rangle$ it is enough to prove the Lemma for $\xi \geq 0, \eta \geq 0$ and $\|\xi\|_{2}=\|\eta\|_{2}=1$. Define $\xi_{z}=\xi^{2 z}$ and $\eta_{z}=\eta^{2-2 z}$ for $\xi(\omega) \neq 0 \neq \eta(\omega), \xi_{z}(\omega)=0$ for $\xi(\omega)=0$; similarly $\eta_{z}(\omega)=0$ for $\eta(\omega)=0$. In particular $\xi_{1 / 2}=\xi$ and $\eta_{1 / 2}=\eta$. Let $z=\delta+i t \in S$ and $p=1 / \delta>1, q=p /(p-1)=1 /(1-\delta)$ the 
conjugate index of $p$; it is easy to see that:

(1) $\xi_{z} \in L^{p}(\Omega),\left\|\xi_{z}\right\|_{p}=1$.

(2) $\eta_{z} \in L^{q}(\Omega),\left\|\eta_{z}\right\|_{q}=1$.

(3) $\left\|\pi_{z}(g) u\right\|_{p}=\|u\|_{p}$ for every $u$ in $L^{p}(\Omega)$ and $g$ in $G$.

Let $\psi(z)=\exp \left(z^{2}-1\right) ;|\psi(z)| \leq 1$ on $S$ and the map $F_{z}=$ $\psi(z)\left\langle\pi_{z}(\cdot) \xi_{z}, \eta_{z}\right\rangle$ is a continuous map on $S$ into $L_{1}^{\infty}(G)$, analytic on $S^{0}$. Since $F_{1 / 2}=\exp \left(-\frac{3}{4}\right)\left\langle\pi_{1 / 2}(\cdot) \xi, \eta\right\rangle$, to prove the Lemma, it suffices to show that:

$$
\int_{\mathbf{R}}\left|\left\langle F_{1 / 2+i t}, u\right\rangle\right|^{2}\left|\phi\left(\frac{1}{2}+i t\right)\right| d t \leq c\|u\|_{2}^{2} \quad \text { for every } u \text { in } C_{00}(G)
$$

and some analytic function $\phi$.

Let $\phi(z)=(r-1) \lg (r-1) /[4 \pi r c(z) c(1-z)]$ where $c(z)$ is the function defined in the preliminaries. $\phi(z)=\phi(z+\pi i / \lg (r-1))$ and so $\phi$ is bounded. Since $\phi(z) \neq 0$ for $\operatorname{Re} z \neq \frac{1}{2}$, it follows that: $|\phi(x+i t)| \geq \min \{|\phi(x+i t)|: t \in \mathbf{R}\}>0$, for every $x \neq \frac{1}{2}, \alpha \leq x \leq \beta$. We have $\left|\phi\left(\frac{1}{2}+i t\right)\right| d t=d m(t)$. Let $J_{k}$ be the interval

$$
J_{k}=[k \pi / \lg (r-1),(k+1) \pi / \lg (r-1)] \quad \text { for } k \in \mathbf{Z} ;
$$

therefore $J_{0}=J$. The functions $\left\|\pi_{1 / 2+i t}(u)\right\|_{H S}$ and $d m(t)$ are periodic; hence, for every $k \in \mathbf{Z}$ :

$$
\int_{J_{k}}\left\|\pi_{1 / 2+i t}(u)\right\|_{H S}^{2} d m(t)=\int_{J}\left\|\pi_{1 / 2+i t}(u)\right\|_{H S}^{2} d m(t) .
$$

Let $h_{k}$ be the maximum of the function

$$
\left|\psi\left(\frac{1}{2}+i t\right)\right|^{2}=\exp \left(-3 / 2-2 t^{2}\right) \text { on } J_{k} \text { and } \sum_{-\infty}^{+\infty} h_{k}=c<+\infty \text {. }
$$

Finally, we have:

$$
\begin{aligned}
\int_{\mathbf{R}}\left|\left\langle F_{1 / 2+i t}, u\right\rangle\right|^{2}\left|\phi\left(\frac{1}{2}+i t\right)\right| d t \\
\quad=\sum_{-\infty}^{+\infty} \int_{J_{k}}\left|\psi\left(\frac{1}{2}+i t\right)\right|^{2}\left|\left\langle\pi_{1 / 2+i t}(u) \xi_{1 / 2+i t}, \eta_{1 / 2+i t}\right\rangle\right|^{2} d m(t) \\
\leq \sum_{-\infty}^{+\infty} h_{k} \int_{J_{k}}\left\|\pi_{1 / 2+i t}(u)\right\|_{H S}^{2} d m(t)=c \int_{J}\left\|\pi_{1 / 2+i t}(u)\right\|_{H S}^{2} d m(t) \\
\leq c\|u\|_{2}^{2},
\end{aligned}
$$

(recall that $\left.\left\|\xi_{1 / 2+i t}\right\|_{2}=\left\|\eta_{1 / 2+i t}\right\|_{2}=1\right)$. 
Proof of Theorem 1. If $G$ acts transitively on $\Omega$, then $\Omega \simeq G / G_{0}$ where $G_{0}$ is the stability subgroup of a fixed point $\omega_{0}$ in $\Omega$. By the "principe de majoration" of C. Herz [14], for every $f$ in $A(G)$ there exists a coefficient of $\pi_{1 / 2}$ such that: $|f(g)| \leq\left\langle\pi_{1 / 2}(g) \xi, \eta\right\rangle$ for every $g$ in $G$. Hence, from Lemma $2, A(G) \subset L^{q}(G)$ for every $q>2$ and $G$ is a Kunze-Stein group.

REMARK. We shall say that a vertex $v$ of a tree is of homogeneity $l$ if $v$ belongs to exactly $l$ edges. Let $X_{l, q}$ be a semihomogeneous tree, that is, a tree such that every vertex is of homogeneity $l$ or $q$ and two adjacent vertices are of homogeneity $l$ and $q$, respectively. We suppose $l \neq q$, otherwise $X$ is a homogeneous tree. Let $S_{l}$ and $S_{q}$ be the subsets of vertices of homogeneity $l$ and $q$, respectively. Theorem 1 is true for semihomogeneous trees, with the same proof.

Indeed, if $G$ is a closed noncompact subgroup of $\operatorname{Aut}\left(X_{l, q}\right)$ acting transitively on the boundary of $X_{l, q}$, then $G \cap K_{v_{0}}$ acts transitively on the boundary for every vertex $v_{0}$. Moreover $G\left(v_{0}\right)=S_{l}$ and $G\left(w_{0}\right)=$ $S_{q}$ for every $v_{0} \in S_{l}$ and $w_{0} \in S_{q}$. Hence, without loss of generality, we can suppose that $l<q$. The representations $\left.\pi_{1 / 2+i t}\right|_{G}$ are irreducible [2, pg. 62] and the Plancherel measure of the Gelfand pair $\left(G, G \cap K_{v_{0}}\right)$ is a multiple of $\left|c\left(\frac{1}{2}+i t\right)\right|^{-2}$ for an analytic function $c(z)$ [10, pg. 153]. The proof proceeds in the same fashion as for homogeneous trees.

4. Simply transitive subgroups. Let $\Gamma$ be a simply transitive subgroup of $\operatorname{Aut}(X)$; for $\eta \in L^{2}(\Omega)$ we define, as in $[11 ; 1]$, the Poisson transform of $\Gamma: \wp(\eta)(x)=\left\langle\pi_{1 / 2}(x) 1, \eta\right\rangle$.

COROLlaRY. $\wp\left(L^{2}(\Omega)\right) \subset l^{q}(\Gamma)$ for every $q>2$.

Proof. By Theorem $1, f(g)=\left\langle\pi_{1 / 2}(g) 1, \eta\right\rangle \in L^{q}(\operatorname{Aut}(X))$ for every $q>2$. Let $\operatorname{Aut}(X)=\Gamma K$ and $g=x k$ with $x \in \Gamma$ and $k \in K$; therefore $\pi_{1 / 2}(g) \mathbf{1}=\pi_{1 / 2}(x) \mathbf{1}$ because $\nu$ is $K$-invariant and so

$$
\wp(\eta)\left\|_{l^{q}(\Gamma)}=\right\| f \|_{L^{q}(\operatorname{Aut}(X))} .
$$

The Corollary follows.

$\Gamma$ is not a Kunze-Stein group (in a discrete Kunze-Stein group every amenable subgroup is finite); nevertheless, we can prove a "weak Kunze-Stein property":

$l_{r}^{p}(\Gamma) *_{\Gamma} l^{2}(\Gamma) \subset l^{2}(\Gamma)$ for every $1<p<2$, where $l_{r}^{p}$ is the space of radial functions in $l^{p}$, that is, the functions which depend only on the length of the words of $\Gamma$ and $*_{\Gamma}$ means the convolution product of 
$\Gamma$. It is easy to see that the "weak Kunze-Stein property" is equivalent to the following: $A_{r}(\Gamma) \subset l^{q}(\Gamma)$ for every $q>2$. This was proved in [5] for free groups on finitely many generators. Notice that $A_{r}(\Gamma)=$ $l_{r}^{2}(\Gamma) *_{\Gamma} l_{r}^{2}(\Gamma)$.

THEOREM 2. The following hold:

(1) $l_{r}^{2}(\Gamma) *_{\Gamma} l^{2}(\Gamma) \subset l^{q}(\Gamma)$ for every $q>2$.

(2) $l^{p}(\Gamma) *_{\Gamma} l_{r}^{2}(\Gamma) \subset l^{2}(\Gamma)$ for every $1<p<2$.

Proof. It is enough to prove (2); (1) follows by duality argument. Putting $\dot{f}(x k)=f(x)$ with $x \in \Gamma$ and $k \in K$, it is possible to identify the functions $f$ on $\Gamma$ with the right $K$-invariant functions $\dot{f}$ on $\operatorname{Aut}(X)=\Gamma K$. The radial functions on $\Gamma$ correspond to the bi $K$ invariant functions on $\operatorname{Aut}(X)$. Let $f \in l^{p}(\Gamma)$ for $1<p<2$ and $\phi \in l_{r}^{2}(\Gamma)$, then the function $\dot{f} * \dot{\phi}$ is right $K$-invariant; hence, by Theorem 1 , the restriction to $\Gamma$ is in $l^{2}(\Gamma)$. Moreover: $\left.(\dot{f} * \dot{\phi})\right|_{\Gamma}=f *_{\Gamma} \phi$ and, from this, Theorem 2 follows.

\section{REFERENCES}

[1] W. Betori and M. Pagliacci, Harmonic analysis for groups acting on trees, Boll. Un. Mat. It., 3-B (1984), 333-349.

[2] F. Bouaziz-Kellil, Representations spheriques des groupes agissant transitivement sur un arbre semi homogène, Thèse de 3-ème cycle, Université de Nancy I.

[3] P. Cartier, Fonctions harmoniques sur un arbre, Symp. Math., 9 (1972), 203270.

[4] F. Choucroun, Groupes opérant simplement transitivement sur un arbre homogène et plongements dans $\mathrm{PGL}_{2}(\kappa)$, C. R. Acad. Sci. Paris, 298 (1984), 313315.

[5] J. M. Cohen and L. deMichele, Radial Fourier-Stieltjes algebra on free groups, Contemporary Math., 10 (Operator algebras and $K$-theory), 1982.

[6] M. Cowling, The Kunze-Stein phenomenon, Ann. of Math., 107 (1978), 209234.

[7] P. Eymard, L'algèbre de Fourier d'un groupe localment compact, Bull. Soc. Math. France, 92 (1964), 181-236.

[8] P. Eymard et N. Lohouè, Sur la racine carrée du noyau de Poisson dans les espaces symétriques et une conjecture de E. M. Stein, Ann. Sci. Ecole Norm. Sup., Ser 4 (8) n. 2 (1975).

[9] J. Faraut, Analyse harmonique sur les paires de Gelfand et les espaces hyperboliques, Analyse harmonique Nancy 1980, CIMPA, Nice (1983).

[10] J. Faraut and M. A. Picardello, The Plancherel measure for symmetric graphs, Ann di Mat. Pura Appl., (IV) 138 (1984), 151-155.

[11] A. Figà-Talamanca and M. A. Picardello, Spherical functions and harmonic analysis on free groups, J. Funct. Anal., 47 (1982), 281-304.

[12] _ Harmonic Analysis on Free Groups, Lecture notes in pure and applied mathematics, Marcel Dekker, New York, 1983. 
[13] C. L. Gulizia, Harmonic analysis of SL(2) over a locally compact field, J. Funct. Anal., 12 (1973), 384-400.

[14] C. Herz, Sur le phénomene de Kunze-Stein, C. R. Acad. Sci. Paris, 271 (1970), 491-493.

[15] R. A. Kunze and E. M. Stein, Uniformly bounded representations and harmonic analysis of the $2 \times 2$ real unimodular group, Amer. J. Math., 82 (1960), 1-62.

[16] S. Lang, $\mathrm{SL}_{2}(\mathbf{R})$, Addison-Wesley, Reading, Mass. 1975.

[17] J. P. Serre, Arbres, amalgames, $\mathrm{SL}_{2}$, Astérisque, 46 (1977).

[18] J. Tits, Sur le groupe des automorphismes d'un arbre, Essays on topology and related topics, Mémoires dédiés a G. de Rham, Springer-Verlag, (1970), 188211.

Received September 1, 1985 and in revised form July 15, 1987. This work was partially supported by G.N.A.F.A. of the C.N.R., Italy.

Università degli Studi di Roma "La SAPIENZa" CitTÀ Universitaria-00187 Roma, Italy 



\section{PACIFIC JOURNAL OF MATHEMATICS}

\section{EDITORS}

V. S. VARADARAJAN

(Managing Editor)

University of California

Los Angeles, CA 90024

Herbert Clemens

University of Utah

Salt Lake City, UT 84112

R. FINN

Stanford University

Stanford, CA 94305
HERMANN FLASCHKA

University of Arizona

Tucson, AZ 85721

RAMESh A. GANGOLLI

University of Washington Seattle, WA 98195

VAUGHAN F. R. JONES

University of California

Berkeley, CA 94720
ROBION KIRBY

University of California

Berkeley, CA 94720

C. C. MOORE

University of California

Berkeley, CA 94720

HAROLD STARK

University of California, San Diego

La Jolla, CA 92093

\section{ASSOCIATE EDITORS}
R. AREnS
E. F. BECKENBACH
B. H. NEUMANN
F. WOLF
K. YOSHIDA (1906-1982)

\section{SUPPORTING INSTITUTIONS}

UNIVERSITY OF ARIZONA

UNIVERSITY OF OREGON

UNIVERSITY OF BRITISH COLUMBIA UNIVERSITY OF SOUTHERN CALIFORNIA

CALIFORNIA INSTITUTE OF TECHNOLOGY

UNIVERSITY OF CALIFORNIA

STANFORD UNIVERSITY

MONTANA STATE UNIVERSITY

UNIVERSITY OF HAWAII

UNIVERSITY OF NEVADA, RENO

UNIVERSITY OF TOKYO

NEW MEXICO STATE UNIVERSITY

UNIVERSITY OF UTAH

OREGON STATE UNIVERSITY

WASHINGTON STATE UNIVERSITY

UNIVERSITY OF WASHINGTON 


\section{Pacific Journal of Mathematics}

\section{Vol. 133, No. $1 \quad$ March, 1988}

John Anderson, Finitely generated algebras and algebras of solutions to partial differential equations $\ldots \ldots \ldots \ldots \ldots \ldots \ldots \ldots \ldots \ldots \ldots \ldots \ldots$

Junichi Aramaki, On an extension of the Ikehara Tauberian theorem . . . . . 13

Giacomo Monti Bragadin, Abstract Riemannian stratifications .......... 31

Lawrence James Brenton and Richard Hill, On the Diophantine equation

$1=\sum 1 / n_{i}+1 / \prod n_{i}$ and a class of homologically trivial complex

surface singularities .................................41

C. Bruce Hughes, Controlled homotopy topological structures $\ldots . \ldots \ldots \ldots 69$

Peter Wilcox Jones and Takafumi Murai, Positive analytic capacity but zero Buffon needle probability . ...........................999

Gary M. Lieberman, Hölder continuity of the gradient at a corner for the

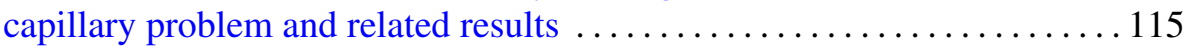

Feng Luo, Representing homology classes of $C \mathbf{P}^{2} \overline{C \mathbf{P}^{2}}$

Claudio Nebbia, Groups of isometries of a tree and the Kunze-Stein

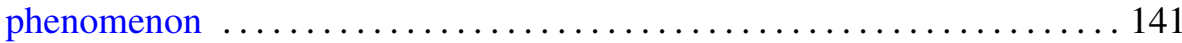

Stefan Richter, Unitary equivalence of invariant subspaces of Bergman and

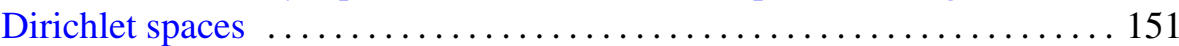

Paul Frederick Ringseth, The Selberg trace formula for groups without Eisenstein series ..................................... 157

Abderrazzak Sersouri, The Mazur property for compact sets $\ldots \ldots \ldots \ldots 185$ Alladi Sitaram, On an analogue of the Wiener Tauberian theorem for symmetric spaces of the noncompact type 